\title{
Towards endoscopic augmented reality for robotically assisted minimally invasive cardiac surgery*
}

\author{
Frédéric Devernay, Fabien Mourgues, Ève Coste-Manière \\ CHIR team, InRIA, 2004 route des Lucioles, BP93, 06902 Sophia Antipolis Cedex, France
}

\begin{abstract}
One of the problems tightly linked to endoscopic surgery is the fact that, because of the narrow field of view, it is sometimes quite difficult to locate the objects that can be seen through the endoscope. This is especially true in cardiac surgery, where it is difficult to not confuse two coronary arteries on a beating heart. We propose in this paper a methodology to achieve coronary localisation by augmented reality on a robotized stereoscopic endoscope.

The method we propose involves five steps: making a time-variant $3 D$ model of the beating heart using coronarography and CT-scan or MRI, calibrating the stereoscopic endoscope, reconstructing the $3 D$ operating field, registering the operating field surface with the $3 D$ heart model, and adding information on the endoscopic images by augmented reality.

The da Vinci ${ }^{T M}$ surgical system was used for our first experiments with the Cardiac Surgery team at Hôpital Européen Georges Pompidou, Paris, France.
\end{abstract}

\section{Introduction}

The introduction of robots in cardio-vascular surgery came from the limitations imposed on the surgeon by manually controlled minimally invasive surgery (MIS) instruments. Namely, the surgeon finds his movements, vision and tactile sensing reduced and severely altered [13, 2]. The use of a robotic manipulator can remedy the loss of dexterity by incorporating additional degrees of freedom at the end of the tools, as is the case with the EndoWrist ${ }^{\mathrm{TM}}$ system of the da Vinci ${ }^{\mathrm{TM}}$ robot (see [11] for details about the system). In addition, a robotic system offers an increased precision and stability of the movement.

\footnotetext{
*Thanks to the following persons and institutions for providing data for our experiments and feedback: Alain Carpentier, Renaud Séverac-Bastide, Didier Blanchard, Hôpital Européen Georges Pompidou; Régis Vaillant, GE Medical Systems; Kenneth Salisbury, Gary Guthart, Intuitive Surgical.
}

However, minimally invasive surgery brings its own limitations and problems. First, since the operating field will be reached through a small set of incision sites (at least three in the case the da Vinci system), these incisions (or ports) must be carefully chosen to satisfy at least a few constraints: the whole operating field must be reachable, the instruments must be positioned in a natural way (left instrument on the left, right instrument on the right, and endoscope above both), and the robot must be able to deliver the right forces to the instruments during the whole intervention. An optimised port-placement solution [4] takes all theses constraints into account, and computes the location of incisions, given the anatomy of a given patient as extracted from MRI or CT-scan.

Another problem comes from the fact that the field of view of the endoscope is restricted, and the surgeon has a keyhole view of the operating field and its surrounding. In the case of cardiac surgery, this is particularly sensible: during the first phase of the operation, when the surgeon locates the landmarks necessary for the operation (such as the location of the coronary and the position of the stenosis on the coronary) the targets are mostly hidden by the pericardium and the heart is still beating. During the execution of the operation, the coronaries may be partly hidden e.g. by fat or the inter-ventricular groove. For these reasons, the narrow field of view may lead to misidentifying the coronary (e.g. taking the circumflex for the inter-ventricular) or the stenosis area.

To solve this issue, we propose to add "cartographic" information on the endoscopic view, by indicating the position of the coronaries with respect to the field of view. To achieve this, having the 3D anatomical model of the patient extracted from MRI or CT-scan and the position of the endoscope with respect to the patient is not sufficient, since the organs (in particular the lungs and the heart) are displaced by the inflated gas. Besides, these image modalities cannot model a beating heart: they give at most a 3D model at a given heart time (in the case of gated MRI or CT), and do not give the full time-varying $(3 \mathrm{D}+\mathrm{t})$ model.

A few systems have been proposed for augmented reality based on 3D laparoscopy [3, 10], but none addresses 
the problem of recovering the position of deformable or time-varying organs such as the heart, and none is designed specifically for robotically-assisted surgery.

The proposed solution consists of first making a $3 \mathrm{D}+\mathrm{t}$ heart model by using information extracted from both standard coronarography sequences and MRI or CT-scan data. During the operation, this $3 \mathrm{D}+\mathrm{t}$ model has to be registered with respect to the stereoscopic endoscope. Since this is a robotic system, we can easily get the global position of the endoscope within the body, but the terminal optics still have to be calibrated for further image processing.

The real-time image processing loop consists of reconstructing the observed 3D surfaces using stereoscopy, removing instruments from this reconstruction, incrementally building a global 3D surface model by fusion of individual surface patches, and registering the resulting surface with the $3 \mathrm{D}+\mathrm{t}$ deformable organ model to recover its position. Informations about the location of coronary arteries with respect to the operating field can then be superimposed on the stereoscopic endoscopic views.

This is still work-in-progress: The various components of the application presented below have come to various degrees of achievement and are presented in their current state.

\section{Modelling a 3D Beating Heart}

The 3D+t heart modelling method is based on widely available data: the coronary tree is extracted from two or more standard coronarography sequences, and the epicardium surface is computed from 3D MRI or CT-scan data of the heart.

The $3 \mathrm{D}+\mathrm{t}$ coronary tree is extracted in three steps [14] as described in the following.

\subsection{Coronaries central lines detection}

First, every 2D images are processed with a multi-scale model based analysis. The result of this stage is a central lines magnitude, a map of the directions and scale of detection of the presumed vessels for each $2 \mathrm{D}$ image. Then, the 2D coronary tree is extracted and labelled semiautomatically from the first image of each sequence (corresponding to different incidences) with an interactive tool near of the Intelligent Scissors and based on the previous maps.

\subsection{Tracking vessels over image sequences}

The extracted and labelled tree is automatically tracked over all images of each sequence using B-snakes with an image energy deduced from the multi-scale response. The
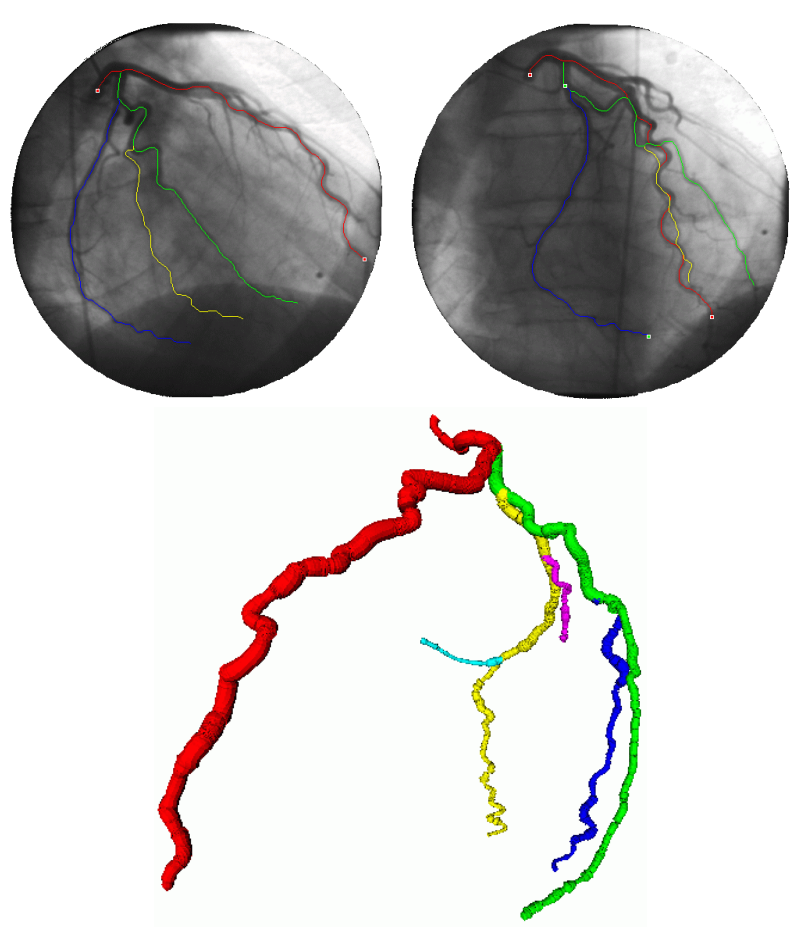

Figure 1. Two views from the coronarography sequences, and the reconstructed 3D coronary tree

optimisation is performed in two steps to prevent big shifts, crossing and overlapping of arteries.

\subsection{D reconstruction of the coronary tree}

A 3D skeletized model is computed for each cardiac time by using stereoscopy on extracted centrelines. The nonsimultaneity of the acquisitions induces a 3D heart shift between the projections due to the patient's motion and respiration. This problem is solved by matching point to point the globally matched centrelines with weak epipolar constraints and a priori constraints on the matching of the junctions and bifurcations. Then $3 \mathrm{D}$ centrelines are reconstructed and the sensors parameters are optimised with bundle adjustment technic (Figure 11). 3D sections of arteries are estimated from the scale deduced from the multi-scale preprocessing.

\subsection{Full 3D+t heart model}

The $3 \mathrm{D}+\mathrm{t}$ coronary tree must then be fused with the $3 \mathrm{D}$ epicardium surface extracted from gated MRI or CT-scan to build a full $3 \mathrm{D}+\mathrm{t}$ heart model with all the coronary arteries. 


\section{Calibration of the Stereoscopic Endoscope}

The calibration of a stereoscopic endoscope involves methods that are well established. The first kind of calibration method uses a calibration grid to compute the internal and external camera parameters [15].

Another kind of method, called self-calibration, first extracts the epipolar geometry of the stereoscopic system, contained in the fundamental matrix, which enables a 3D reconstruction of the observed scene up to a $3 \mathrm{D}$ projective transformation [6]. Using several stereo pairs of the same scene with various positions of the stereoscopic endoscope, one can then recover the full Euclidean geometry of the 3D scene up to a scale factor [12, 5].

All these methods compute the position of the cameras with respect to world coordinates (defined either by the calibration grid or arbitrarily), but we need to find their position with respect to the robot arm (and eventually recover the scale factor on the world in the case of self-calibration). This can be easily done by doing camera calibration with various positions of the robot arm and computing the fixed displacement between the cameras and the arm.

\section{Operating Field Surface Reconstruction by Stereoscopy}

The operating field as seen through the stereoscopic endoscope is reconstructed using correlation-based stereo (Figure 2). The goal is to reconstruct the organs surface, and then register it with organs models to compute their respective positions and deformations. A parallel implementation of the stereoscopic matching algorithm was developed [7] and ported to PVM (Parallel Virtual Machine) for better scalability: in PVM, any number of nodes can be used, and the same code can run either on a multi-processor architecture or on a (possibly heterogeneous) cluster of workstations.

Since we want to extract the 3D surface of the organs, the instruments have to be removed from this reconstruction. We could use the position of the instrument computed from the robot coordinates, and remove these instruments by using their a priori position in the images, but we preferred a more general technique which applies even if there are manually-controlled instruments in the operating field (such as a stabiliser), or if the instrument localisation obtained from the robot's articular coordinates is not accurate enough.

For each position of the endoscope, the 3D scene viewed by the cameras is reconstructed from the stereo pair. These reconstructions are considered as the input measures of a learning process: the z-position of each point on the organ surface is individually modelled as a Gaussian distribution (mean and standard deviation). The $\mathrm{z}$-values obtained from
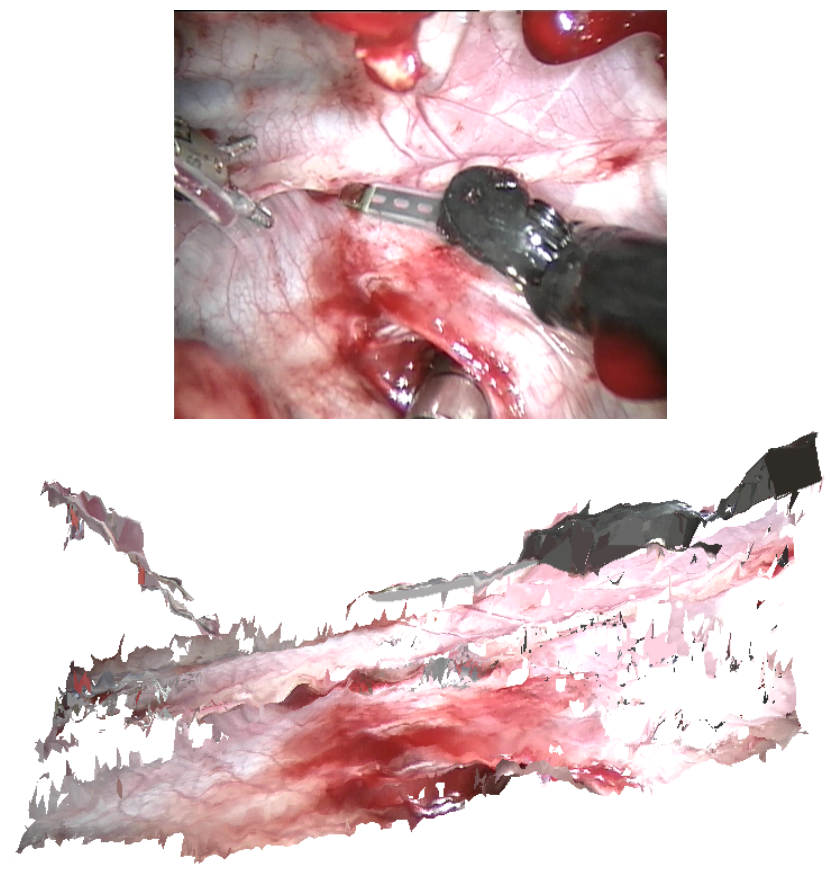

Figure 2. Left view from the stereoscopic endoscope (top) and perspective view of the reconstruction showing the instruments above the operating field (bottom)

each new stereo pair are classified according to the current $\mathrm{z}$-statistics of the corresponding organ vertex. The z-value is integrated in the organ model only if it's either within the standard deviation of the corresponding model point, or if it's farther from the observer. This way, instruments that were inside the operating field at the beginning of the acquisition can easily be removed from the reconstruction. Everything that is not classified as part of the organ model is considered foreground.

These small organ surface patches - for each endoscopic position - are then fused to construct a larger surface of the organ, which can then be used for a better registration. The motion of the endoscope is computed both from the robot's articular coordinated and from stereo tracking of points of interest.

The main issue to solve for this stage is to find an appropriate representation and 2-dimensional parameterisation of the surface, to reduce the complexity of the data fusion problem. Tor the time being, we use a reference frame defined by the first position of the endoscope. 


\section{Real-Time Surface Registration}

In this step, the surface reconstructed by stereoscopy has to be registered with the surface extracted by segmentation of the MRI or CT-scan data. Since the organs we consider are highly deformable and time-varying, we use a locally affine registration method based on ICP (Iterated Closest Point) [1, 8].

A parallel implementation of the ICP algorithm is being developed using PVM. This implementation takes into account the fact that the reference data (the organ model in our case) is fixed and known, so that some of the data structures used by the algorithm (e.g. the $k$-D tree used for closest point computation) can be precomputed and shared between tasks. If the surgeon interacts with the organs and modifies their configuration or topology, these data structures have to be updated to reflect the changes.

\section{Augmented Reality}

Once the observed surface is registered with the $3 \mathrm{D}+\mathrm{t}$ heart model, the cartographic informations about the location of coronary arteries can be superimposed on the stereoscopic video images. If the arteries are outside of field of view, these informations can be directions to the target area, and when the arteries are inside the field of view, they can be superimposed on the stereo images. Since there are also instruments present in the field of view, the method should deal with occlusions by the instruments, which is easy since we already know where they are from the operating field surface reconstruction and background vs. foreground classification.

One of the problem that arises comes from the fact that we have to overlay data obtained for images acquired some time ago over live images: we hope to get the whole image processing loop (operating field reconstruction and registration) runiing at around $1 \mathrm{~Hz}$, so that we may have to overlay data that is around one second old. The turnaround is to simply have a video overlay loop that runs at video rate, and compensate for endoscope and organ motion. The endoscope motion can be obtained from the robot's articular coordinates, and the organ motion can be estimated using external measurements (respiratory and electro-cardiogram data in the case of the heart). All these data can be easily acquired or computed at video rate.

Whenever a new registration result is obtained, it is used as the new starting point for overlay position estimation, using the date of stereo pair acquisition as the starting date of the acquisition. Consequently, though the image processing loop does not run at video rate, overlay information can still be consistent with the instantaneous video images.

\section{Discussion and Conclusions}

We described in this paper a set of algorithms that once put together will lead to an augmented reality system for robotically assisted minimally invasive surgery. Some of the individual methods were readily available from past developments, such as the calibration of the endoscope or correlation-based stereo, but new algorithms are also being developed to solve some of the problems encountered during this project (such as the $3 \mathrm{D}+\mathrm{t}$ heart modelling or the background vs. foreground separation).

The different steps of the application bring various levels of difficulty, and are at various levels of completion. While the solutions to endoscope calibration and $3 \mathrm{D}+\mathrm{t}$ organ modeling were clearly identified, the problems related to the real-time image processing loop, which includes stereoscopy, 3D surface reconstruction, foreground elimination, and registration), will probably be the hardest part to achieve.

Whereas this work is application-driven, we try to make these new algorithms as generic as possible, so that they can be used in other augmented reality applications or in other application fields: For example, the background vs. foreground separation method makes no assumption about the knowledge of instruments position so that it can be used in other contexts, and the stereo-based operating field reconstruction method is also being used for augmented virtuality and brain-shift estimation in neurosurgery [9].

Another subject of research pursued within the CHIR team at INRIA concerns the safe integration of these algorithms into a real-time architecture : all these algorithms work at different rates, exchange different kind of data, and have to be integrated seamlessly with the da Vinci robotic system or any other robotic surgical system.

\section{References}

[1] P. J. Besl and N. D. McKay. A method for registration of 3-D shapes. IEEE Transactions on Pattern Analysis and Machine Intelligence, 14(2):239-256, Feb. 1992.

[2] G. B. Cadière and J. Leroy. Principes généraux de la chirurgie laparoscopique. Encycl. Méd. Chir. (Techniques chirurgicales - Appareil digestif), volume 40, page 9. Elsevier-Paris, 1999.

[3] J. Cortadellas, G. Bellaire, and G. Grasche. New concepts for intraoperative navigation: calibration of a 3-D laparoscope. In BVM'2000 - Bildverarbeitung für die Medizin, pages 158-162, Munich, Germany, 2000. Springer Verlag.

[4] È. Coste-Manière, L. Adhami, R. Severac-Bastide, K. Salisbury, J.-D. Boissonnat, N. Swarup, G. Guthart, E. Mousseaux, D. Blanchard, and A. Carpentier. Optimized port placement for the totally endoscopic coronary artery bypass grafting using the da vinci robotic system. Lecture 
Notes in Control and Information, ISEROO Intl. Symposium on Experimental Robotics, 2001.

[5] F. Devernay and O. Faugeras. From projective to euclidean reconstruction. In Proceedings of the International Conference on Computer Vision and Pattern Recognition, pages 264-269, San Francisco, CA, June 1996. IEEE.

[6] O. Faugeras. Three-Dimensional Computer Vision: a Geometric Viewpoint. MIT Press, 1993.

[7] O. Faugeras, B. Hotz, H. Mathieu, T. Viéville, Z. Zhang, P. Fua, E. Théron, L. Moll, G. Berry, J. Vuillemin, P. Bertin, and C. Proy. Real time correlation based stereo: algorithm implementations and applications. Technical Report 2013, INRIA Sophia-Antipolis, France, 1993.

[8] J. Feldmar and N. Ayache. Rigid, affine and locally affine registration of free-form surfaces. Intl. Jour. Comp. Vision, 18(2):99-119, May 1996.

[9] O. Fleig, F. Devernay, J.-M. Scarabin, and P. Jannin. Surface reconstruction of the surgical field from stereoscopic microscope views in neurosurgery. In Proc. CARS'2001 (Computer Assisted Radiology and Surgery), Berlin, 2001.

[10] H. Fuchs, M. Livingston, R. Raskar, D. Colucci, K. Keller, A. State, J. Crawford, P. Rademacher, S. Drake, and A.Meyer. Augmented reality visualization for laproscopic surgery. In Proc. of Medical Image Computing and Computer-Assisted Intervention '98 (MICCAI'98), volume 1496 of $L N C S$, pages 934-943. Springer, 1998.

[11] G. Guthart and J. K. Salisbury Jr. The intuitive telesurgery system: Overview and application. In Proceedings of the 2000 IEEE International Conference on Robotics and Automation, pages 618-622, San Francisco, CA, 2000.

[12] R. Horaud and G. Csurka. Self-calibration and euclidean reconstruction using motions of a stereo rig. In Proceedings of the 6th International Conference on Computer Vision, Bombay, India, Jan. 1998. IEEE Computer Society, IEEE Computer Society Press.

[13] D. Loulmet, A. Carpentier, N. d'Attellis, A. Berrebi, C. Cardon, O. Ponzio, B. Aupècle, and J. Y. M. Relland. Endoscopic coronary artery bypass grafting with the aid of robotic assisted instruments. The journal of thoracic and cardiovascular surgery, 118(1), July 1999.

[14] F. Mourgues, F. Devernay, G. Malandain, and E. CosteManière. Building a 3D+t model of the coronary tree from coronarography sequences. In Journées Orasis 2001, Cahors, France, 2001. in French.

[15] R. Y. Tsai. A versatile camera calibration technique for highaccuracy 3D machine vision metrology using off-the-shelf TV cameras and lenses. IEEE Journal of Robotics and Automation, 3(4):323-344, Aug. 1987. 